\title{
On a new Hilbert-type integral inequality involving the upper limit functions
}

Hongmin $\mathrm{Mo}^{1 *}$ and Bicheng Yang ${ }^{2}$

"Correspondence:

mohongmin1969@163.com

${ }^{1}$ College of Mathematics and

Statistics, Jishou University, Jishou,

P.R. China

Full list of author information is

available at the end of the article

\begin{abstract}
By applying the weight functions and the idea of introduced parameters we give a new Hilbert-type integral inequality involving the upper limit functions and the beta and gamma functions. We consider equivalent statements of the best possible constant factor related to a few parameters. As applications, we obtain a corollary in the case of a nonhomogeneous kernel and some particular inequalities.
\end{abstract}

MSC: 26D15

Keywords: Weight function; Hilbert-type integral inequality; Upper limit function; Parameter; Beta function; Gamma function

\section{Introduction}

If $0<\sum_{m=1}^{\infty} a_{m}^{2}<\infty$ and $0<\sum_{n=1}^{\infty} b_{n}^{2}<\infty$, then we have the following discrete Hilbert inequality with the best possible constant factor $\pi$ ([1], Theorem 315):

$$
\sum_{m=1}^{\infty} \sum_{n=1}^{\infty} \frac{a_{m} b_{n}}{m+n}<\pi\left(\sum_{m=1}^{\infty} a_{m}^{2} \sum_{n=1}^{\infty} b_{n}^{2}\right)^{1 / 2} .
$$

Assuming that $0<\int_{0}^{\infty} f^{2}(x) d x<\infty$ and $0<\int_{0}^{\infty} g^{2}(y) d y<\infty$, we still have the following integral analogue of (1) ([1], Theorem 316):

$$
\int_{0}^{\infty} \int_{0}^{\infty} \frac{f(x) g(y)}{x+y} d x d y<\pi\left(\int_{0}^{\infty} f^{2}(x) d x \int_{0}^{\infty} g^{2}(y) d y\right)^{1 / 2}
$$

where the constant factor $\pi$ is the best possible. Inequalities (1) and (2) are playing an important role in analysis and its applications [2-13].

The following half-discrete Hilbert-type inequality was provided in 1934 ([1], Theorem 351): If $K(x)(x>0)$ is a decreasing function, $p>1, \frac{1}{p}+\frac{1}{q}=1,0<\phi(s)=\int_{0}^{\infty} K(x) x^{s-1} d x<\infty$, $f(x) \geq 0$, and $0<\int_{0}^{\infty} f^{p}(x) d x<\infty$, then

$$
\sum_{n=1}^{\infty} n^{p-2}\left(\int_{0}^{\infty} K(n x) f(x) d x\right)^{p}<\phi^{p}\left(\frac{1}{q}\right) \int_{0}^{\infty} f^{p}(x) d x .
$$

(c) The Author(s) 2020. This article is licensed under a Creative Commons Attribution 4.0 International License, which permits use sharing, adaptation, distribution and reproduction in any medium or format, as long as you give appropriate credit to the original author(s) and the source, provide a link to the Creative Commons licence, and indicate if changes were made. The images or other third party material in this article are included in the article's Creative Commons licence, unless indicated otherwise in a credit line to the material. If material is not included in the article's Creative Commons licence and your intended use is not permitted by statutory regulation or exceeds the permitted use, you will need to obtain permission directly from the copyright holder. To view a copy of this licence, visit http://creativecommons.org/licenses/by/4.0/. 
In recent years, some new extensions of (3) were given by [14-19].

In 2006, using the Euler-Maclaurin summation formula, Krnic et al. [20] gave an extension of (1) with the kernel $\frac{1}{(m+n)^{\lambda}}(0<\lambda \leq 14)$. In 2019, following [20], Adiyasuren et al. [21] considered an extension of (1) involving the partial sums. In 2016-2017, by applying the weight functions Hong $[22,23]$ considered some equivalent statements of the extensions of (1) and (2) with a few parameters. Some similar works were provided in [24-26].

In this paper, following [21, 22], by the use of the weight functions and the idea of introduced parameters, we give a new Hilbert-type integral inequality with the kernel $\frac{1}{(x+y)^{\lambda}}$ $(\lambda>0)$ involving the upper limit functions and the beta and gamma functions. We consider the equivalent statements of the best possible constant factor related to a few parameters. As applications, we obtain a corollary in the case of nonhomogeneous kernel and some particular inequalities.

\section{Some lemmas}

In what follows, we assume that $p>1, \frac{1}{p}+\frac{1}{q}=1, \lambda>0, \lambda_{1}, \lambda_{2} \in(0, \lambda+1), f(x)$ and $g(y)$ are nonnegative measurable functions in $R_{+}=(0, \infty), f(x)=o\left(e^{x}\right), g(y)=o\left(e^{y}\right)(x, y \rightarrow \infty)$, such that for any $A=(0, a)(a>0), f, g \in L^{1}(A)$, and the upper limit functions are defined by

$$
F(x):=\int_{0}^{x} f(t) d t \quad(x \geq 0) \quad \text { and } \quad G(y):=\int_{0}^{y} g(t) d t \quad(y \geq 0) \text {, }
$$

satisfying

$$
0<\int_{0}^{\infty} x^{-p \lambda_{1}-\left(\lambda-\lambda_{1}-\lambda_{2}\right)-1} F^{p}(x) d x<\infty \quad \text { and } \quad 0<\int_{0}^{\infty} y^{-q \lambda_{2}-\left(\lambda-\lambda_{1}-\lambda_{2}\right)-1} G^{q}(y) d y<\infty
$$

By the definition of the gamma function, for $\lambda, x, y>0$, the following expression holds:

$$
\frac{1}{(x+y)^{\lambda}}=\frac{1}{\Gamma(\lambda)} \int_{0}^{\infty} t^{\lambda-1} e^{-(x+y) t} d t
$$

Lemma 1 For $t>0$, we have the following expressions:

$$
\begin{aligned}
& \int_{0}^{\infty} e^{-t x} f(x) d x=t \int_{0}^{\infty} e^{-t x} F(x) d x \\
& \int_{0}^{\infty} e^{-t y} g(y) d y=t \int_{0}^{\infty} e^{-t y} G(y) d y
\end{aligned}
$$

Proof We find

$$
\begin{aligned}
\int_{0}^{\infty} e^{-t x} f(x) d x & =\int_{0}^{\infty} e^{-t x} d F(x) \\
& =\left.e^{-t x} F(x)\right|_{0} ^{\infty}-\int_{0}^{\infty} F(x) d e^{-t x} \\
& =\lim _{x \rightarrow \infty} \frac{F(x)}{e^{t x}}+t \int_{0}^{\infty} e^{-t x} F(x) d x
\end{aligned}
$$


If $F(\infty)=$ constant, then $\lim _{x \rightarrow \infty} \frac{F(x)}{e^{t x}}=0$, and (5) follows; if $F(\infty)=\infty$, in view of $f(x)=$ $o\left(e^{x}\right)(x \rightarrow \infty)$, we find

$$
\begin{aligned}
\int_{0}^{\infty} e^{-t x} f(x) d x & =\lim _{x \rightarrow \infty} \frac{F^{\prime}(x)}{\left(e^{t x}\right)_{x}^{\prime}}+t \int_{0}^{\infty} e^{-t x} F(x) d x \\
& =\lim _{x \rightarrow \infty} \frac{f(x)}{t e^{t x}}+t \int_{0}^{\infty} e^{-t x} F(x) d x \\
& =0+t \int_{0}^{\infty} e^{-t x} F(x) d x
\end{aligned}
$$

and then (5) follows. In the same way, we have (6).

The lemma is proved.

Lemma 2 For $s>0, \mu, \sigma \in(0, s)$, define the following weight functions:

$$
\begin{aligned}
& \varpi(\sigma, x):=x^{s-\sigma} \int_{0}^{\infty} \frac{t^{\sigma-1}}{(x+t)^{s}} d t \quad\left(x \in \mathrm{R}_{+}\right), \\
& \omega(\mu, y):=y^{s-\mu} \int_{0}^{\infty} \frac{t^{\mu-1}}{(t+y)^{s}} d t \quad\left(y \in \mathrm{R}_{+}\right) .
\end{aligned}
$$

We have the following expressions:

$$
\begin{array}{ll}
\varpi(\sigma, x)=B(\sigma, s-\sigma) & \left(x \in \mathrm{R}_{+}\right), \\
\omega(\mu, y)=B(\mu, s-\mu) & \left(y \in \mathrm{R}_{+}\right),
\end{array}
$$

where $B(u, v):=\int_{0}^{\infty} \frac{t^{u-1}}{(1+t)^{u+v}} d t(u, v>0)$ is the beta function satisfying

$$
B(u, v)=\frac{1}{\Gamma(u+v)} \Gamma(u) \Gamma(v)
$$

Proof Setting $u=\frac{t}{x}$, we find

$$
\varpi(\sigma, x)=x^{s-\sigma} \int_{0}^{\infty} \frac{(u x)^{\sigma-1}}{(x+u x)^{s}} x d u=\int_{0}^{\infty} \frac{u^{\sigma-1}}{(1+u)^{s}} d u=B \quad(\sigma, s-\sigma),
$$

namely, (9) follows. In the same way, we have (10).

The lemma is proved.

Lemma 3 Suppose that $s>0, \mu, \sigma \in(0, s)$. We have the following inequality:

$$
\begin{aligned}
& \int_{0}^{\infty} \int_{0}^{\infty} \frac{f(x) g(y)}{(x+y)^{s}} d x \leq B^{\frac{1}{p}}(\sigma, s-\sigma) B^{\frac{1}{q}}(\mu, s-\mu) \\
& \quad \times\left[\int_{0}^{\infty} x^{p(1-\mu)-(s-\mu-\sigma)-1} f^{p}(x) d x\right]^{\frac{1}{p}}\left[\int_{0}^{\infty} y^{q(1-\sigma)-(s-\mu-\sigma)-1} g^{q}(y) d y\right]^{\frac{1}{q}} .
\end{aligned}
$$


For $\lambda>0, s=\lambda+2(>2), \lambda_{1}=\mu-1 \in(0, \lambda+1), \lambda_{2}=\sigma-1 \in(0, \lambda+1)$, by the substitution $f(x)=F(x)$ and $g(y)=G(y)$ in (11) we can reduce it to the following:

$$
\begin{aligned}
& \int_{0}^{\infty} \int_{0}^{\infty} \frac{F(x) G(y)}{(x+y)^{\lambda+2}} d x d y<B^{\frac{1}{p}}\left(\lambda_{2}+1, \lambda+1-\lambda_{2}\right) B^{\frac{1}{q}}\left(\lambda_{1}+1, \lambda+1-\lambda_{1}\right) \\
& \quad \times\left[\int_{0}^{\infty} x^{-p \lambda_{1}-\left(\lambda-\lambda_{1}-\lambda_{2}\right)-1} F^{p}(x) d x\right]^{\frac{1}{p}}\left[\int_{0}^{\infty} y^{-q \lambda_{2}-\left(\lambda-\lambda_{1}-\lambda_{2}\right)-1} G^{q}(y) d y\right]^{\frac{1}{q}} .
\end{aligned}
$$

Proof By Hölder's inequality (see [27]) we obtain

$$
\begin{aligned}
\int_{0}^{\infty} & \int_{0}^{\infty} \frac{f(x) g(y)}{(x+y)^{s}} d x d y=\int_{0}^{\infty} \int_{0}^{\infty} \frac{1}{(x+y)^{s}}\left[\frac{y^{(\sigma-1) / p}}{x^{(\mu-1) / q}} f(x)\right]\left[\frac{x^{(\mu-1) / q}}{y^{(\sigma-1) / p}} g(y)\right] d x d y \\
\leq & \left\{\int_{0}^{\infty}\left[\int_{0}^{\infty} \frac{1}{(x+y)^{s}} \frac{y^{\sigma-1} d y}{x^{(\mu-1)(p-1)}}\right] f^{p}(x) d x\right\}^{\frac{1}{p}} \\
& \times\left\{\int_{0}^{\infty}\left[\int_{0}^{\infty} \frac{1}{(x+y)^{s}} \frac{x^{\mu-1} d x}{y^{(\sigma-1)(q-1)}}\right] g^{q}(y) d y\right\}^{\frac{1}{q}} \\
= & {\left[\int_{0}^{\infty} \omega(\sigma, x) x^{p(1-\mu)-(\lambda-\mu-\sigma)-1} f^{p}(x) d x\right]^{\frac{1}{p}} } \\
& \times\left[\int_{0}^{\infty} \omega(\mu, y) y^{q(1-\sigma)-(\lambda-\mu-\sigma)-1} g^{q}(y) d y\right]^{\frac{1}{q}} .
\end{aligned}
$$

Then by (9) and (10) we have (11).

By simplifications of (11) we have

$$
\begin{gathered}
\int_{0}^{\infty} \int_{0}^{\infty} \frac{F(x) G(y)}{(x+y)^{\lambda+2}} d x d y \leq B^{\frac{1}{p}}\left(\lambda_{2}+1, \lambda+1-\lambda_{2}\right) B^{\frac{1}{q}}\left(\lambda_{1}+1, \lambda+1-\lambda_{1}\right) \\
\times\left[\int_{0}^{\infty} x^{-p \lambda_{1}-\left(\lambda-\lambda_{1}-\lambda_{2}\right)-1} F^{p}(x) d x\right]^{\frac{1}{p}}\left[\int_{0}^{\infty} y^{-q \lambda_{2}-\left(\lambda-\lambda_{1}-\lambda_{2}\right)-1} G^{q}(y) d y\right]^{\frac{1}{q}} .
\end{gathered}
$$

If (14) keeps the form of equality, then, in view of the proof of (13), there exist constants $A$ and $B$ such that they are not all zero, satisfying for $s=\lambda+2, \lambda_{1}=\mu-1, \lambda_{2}=\sigma-1$,

$$
\begin{aligned}
& A x^{-p \lambda_{1}-\left(\lambda-\lambda_{1}-\lambda_{2}\right)-1} x^{\left(\lambda-\lambda_{1}-\lambda_{2}\right)+1} F^{p}(x) \\
& =B y^{-q \lambda_{2}} G^{q}(y) \quad \text { a.e. in }(0, \infty) \times(0, \infty) .
\end{aligned}
$$

Without loss of generality, we assume that $A \neq 0$. Then for fixed $y \in(0, \infty)$, we have

$$
x^{-p \lambda_{1}-\left(\lambda-\lambda_{1}-\lambda_{2}\right)-1} F^{p}(x)=\left(\frac{B}{A} y^{-q \lambda_{2}} G^{q}(y)\right) x^{-1-\left(\lambda-\lambda_{1}-\lambda_{2}\right)} \quad \text { a.e. in }(0, \infty),
$$

which contradicts the fact that

$$
0<\int_{0}^{\infty} x^{-p \lambda_{1}-\left(\lambda-\lambda_{1}-\lambda_{2}\right)-1} F^{p}(x) d x<\infty
$$

since for any $\lambda-\lambda_{1}-\lambda_{2} \in \mathbf{R}, \int_{0}^{\infty} x^{-1-\left(\lambda-\lambda_{1}-\lambda_{2}\right)} d x=\infty$. Therefore inequality (12) follows.

The lemma is proved. 


\section{Main results}

Theorem 1 We have the following inequality:

$$
\begin{aligned}
I:= & \int_{0}^{\infty} \int_{0}^{\infty} \frac{f(x) g(y)}{(x+y)^{\lambda}} d x d y<\frac{\Gamma(\lambda+2)}{\Gamma(\lambda)} B^{\frac{1}{p}}\left(\lambda_{2}+1, \lambda+1-\lambda_{2}\right) B^{\frac{1}{q}}\left(\lambda_{1}+1, \lambda+1-\lambda_{1}\right) \\
& \times\left[\int_{0}^{\infty} x^{-p \lambda_{1}-\left(\lambda-\lambda_{1}-\lambda_{2}\right)-1} F^{p}(x) d x\right]^{\frac{1}{p}}\left[\int_{0}^{\infty} y^{-q \lambda_{2}-\left(\lambda-\lambda_{1}-\lambda_{2}\right)-1} G^{q}(y) d y\right]^{\frac{1}{q}} .
\end{aligned}
$$

In particular, for $\lambda_{1}+\lambda_{2}=\lambda\left(\lambda_{1}, \lambda_{2} \in(0, \lambda)\right)$, we reduce it to the following inequality:

$$
\begin{aligned}
\int_{0}^{\infty} \int_{0}^{\infty} \frac{f(x) g(y)}{(x+y)^{\lambda}} d x d y< & \lambda_{1} \lambda_{2} B\left(\lambda_{1}, \lambda_{2}\right)\left(\int_{0}^{\infty} x^{-p \lambda_{1}-1} F^{p}(x) d x\right)^{\frac{1}{p}} \\
& \times\left(\int_{0}^{\infty} y^{-q \lambda_{2}-1} G^{q}(y) d y\right)^{\frac{1}{q}}
\end{aligned}
$$

where the constant factor $\lambda_{1} \lambda_{2} B\left(\lambda_{1}, \lambda_{2}\right)$ is the best possible.

Proof Using (4), (5), and (6), we find

$$
\begin{aligned}
I & =\frac{1}{\Gamma(\lambda)} \int_{0}^{\infty} \int_{0}^{\infty} f(x) g(y)\left(\int_{0}^{\infty} t^{\lambda-1} e^{-(x+y) t} d t\right) d x d y \\
& =\frac{1}{\Gamma(\lambda)} \int_{0}^{\infty} t^{\lambda-1}\left(\int_{0}^{\infty} e^{-x t} f(x) d x\right)\left(\int_{0}^{\infty} e^{-y t} g(y) d y\right) d t \\
& =\frac{1}{\Gamma(\lambda)} \int_{0}^{\infty} t^{\lambda+1}\left(\int_{0}^{\infty} e^{-x t} F(x) d x\right)\left(\int_{0}^{\infty} e^{-y t} G(y) d y\right) d t \\
& =\frac{1}{\Gamma(\lambda)} \int_{0}^{\infty} \int_{0}^{\infty} F(x) G(y)\left[\int_{0}^{\infty} t^{\lambda+1} e^{-(x+y) t} d t\right] d x d y \\
& =\frac{\Gamma(\lambda+2)}{\Gamma(\lambda)} \int_{0}^{\infty} \int_{0}^{\infty} \frac{F(x) G(y)}{(x+y)^{\lambda+2}} d x d y .
\end{aligned}
$$

In view of (12), we have (15).

In the case of $\lambda_{1}+\lambda_{2}=\lambda\left(\lambda_{1}, \lambda_{2} \in(0, \lambda)\right)$, we find

$$
\begin{aligned}
& \frac{\Gamma(\lambda+2)}{\Gamma(\lambda)} B^{\frac{1}{p}}\left(\lambda_{2}+1, \lambda+1-\lambda_{2}\right) B^{\frac{1}{q}}\left(\lambda_{1}+1, \lambda+1-\lambda_{1}\right) \\
& =\frac{\Gamma(\lambda+2)}{\Gamma(\lambda)} B^{\frac{1}{p}}\left(\lambda_{2}+1, \lambda_{1}+1\right) B^{\frac{1}{q}}\left(\lambda_{1}+1, \lambda_{2}+1\right) \\
& =\frac{\Gamma(\lambda+2)}{\Gamma(\lambda)} B\left(\lambda_{1}+1, \lambda_{2}+1\right)=\frac{\Gamma(\lambda+2)}{\Gamma(\lambda)} \frac{\Gamma\left(\lambda_{1}+1\right) \Gamma\left(\lambda_{2}+1\right)}{\Gamma(\lambda+2)} \\
& =\lambda_{1} \lambda_{2} \frac{\Gamma\left(\lambda_{1}\right) \Gamma\left(\lambda_{2}\right)}{\Gamma(\lambda)}=\lambda_{1} \lambda_{2} B\left(\lambda_{1}, \lambda_{2}\right),
\end{aligned}
$$

and then (16) follows.

For any $0<\varepsilon<\min \left\{p \lambda_{1}, q \lambda_{2}\right\}$, we set

$$
\tilde{f}(t):=\left\{\begin{array}{ll}
0, & 0<t \leq 1, \\
t^{\lambda_{1}-\frac{\varepsilon}{p}-1}, & t>1,
\end{array} \quad \tilde{g}(t):= \begin{cases}0, & 0<t \leq 1 \\
t^{\lambda_{2}-\frac{\varepsilon}{q}-1}, & t>1 .\end{cases}\right.
$$


We obtain that $\tilde{f}(x)=o\left(e^{x}\right), \tilde{g}(y)=o\left(e^{y}\right)(x, y \rightarrow \infty)$, and $\tilde{F}(x)=\tilde{G}(y) \equiv 0(0<x, y \leq 1)$, where

$$
\begin{aligned}
& \tilde{F}(x)=\int_{0}^{x} \tilde{f}(t) d t=\int_{1}^{x} t^{\lambda_{1}-\frac{\varepsilon}{p}-1} d t=\frac{x^{\lambda_{1}-\frac{\varepsilon}{p}}-1}{\lambda_{1}-\frac{\varepsilon}{p}}<\frac{x^{\lambda_{1}-\frac{\varepsilon}{p}}}{\lambda_{1}-\frac{\varepsilon}{p}} \quad(x>1), \\
& \tilde{G}(y)=\int_{0}^{y} \tilde{g}(t) d t=\int_{1}^{y} t^{\lambda_{2}-\frac{\varepsilon}{q}-1} d t=\frac{y^{\lambda_{2}-\frac{\varepsilon}{q}}-1}{\lambda_{2}-\frac{\varepsilon}{q}}<\frac{y^{\lambda_{2}-\frac{\varepsilon}{q}}}{\lambda_{2}-\frac{\varepsilon}{q}} \quad(y>1) .
\end{aligned}
$$

If there exists a positive constant $M\left(M \leq \lambda_{1} \lambda_{2} B\left(\lambda_{1}, \lambda_{2}\right)\right)$ such that (16) is valid when replacing $\lambda_{1} \lambda_{2} B\left(\lambda_{1}, \lambda_{2}\right)$ by $M$, then, in particular, by substitution of $f(x)=\tilde{f}(x)$ and $g(y)=$ $\tilde{g}(y)$ we have

$$
\tilde{I}:=\int_{0}^{\infty} \int_{0}^{\infty} \frac{\tilde{f}(x) \tilde{g}(y)}{(x+y)^{\lambda}} d x d y<M\left(\int_{0}^{\infty} x^{-p \lambda_{1}-1} \tilde{F}^{p}(x) d x\right)^{\frac{1}{p}}\left(\int_{0}^{\infty} y^{-q \lambda_{2}-1} \tilde{G}^{q}(y) d y\right)^{\frac{1}{q}} .
$$

We find

$$
\begin{aligned}
\tilde{J} & :=\left(\int_{0}^{\infty} x^{-p \lambda_{1}-1} \tilde{F}^{p}(x) d x\right)^{\frac{1}{p}}\left(\int_{0}^{\infty} y^{-q \lambda_{2}-1} \tilde{G}^{q}(y) d y\right)^{\frac{1}{q}} \\
& <\frac{1}{\left(\lambda_{1}-\frac{\varepsilon}{p}\right)\left(\lambda_{2}-\frac{\varepsilon}{q}\right)}\left[\int_{1}^{\infty} x^{-p \lambda_{1}-1}\left(x^{\lambda_{1}-\frac{\varepsilon}{p}}\right)^{p} d x\right]^{\frac{1}{p}}\left[\int_{1}^{\infty} y^{-q \lambda_{2}-1}\left(y^{\lambda_{2}-\frac{\varepsilon}{q}}\right)^{q} d y\right]^{\frac{1}{q}} \\
& =\frac{1}{\left(\lambda_{1}-\frac{\varepsilon}{p}\right)\left(\lambda_{2}-\frac{\varepsilon}{q}\right)}\left(\int_{1}^{\infty} x^{-\varepsilon-1} d x\right)^{\frac{1}{p}}\left(\int_{1}^{\infty} y^{-\varepsilon-1} d y\right)^{\frac{1}{q}} \\
& =\frac{1}{\left(\lambda_{1}-\frac{\varepsilon}{p}\right)\left(\lambda_{2}-\frac{\varepsilon}{q}\right)} \int_{1}^{\infty} x^{-\varepsilon-1} d x=\frac{1}{\varepsilon\left(\lambda_{1}-\frac{\varepsilon}{p}\right)\left(\lambda_{2}-\frac{\varepsilon}{q}\right)} .
\end{aligned}
$$

In view of the Fubini theorem (see [28]), it follows that

$$
\begin{aligned}
\tilde{I} & =\int_{1}^{\infty}\left[\int_{1}^{\infty} \frac{y^{\lambda_{2}-\frac{\varepsilon}{q}-1}}{(x+y)^{\lambda}} d y\right] x^{\lambda_{1}-\frac{\varepsilon}{p}-1} d x=\int_{1}^{\infty} x^{-\varepsilon-1}\left[\int_{1 / x}^{\infty} \frac{u^{\lambda_{2}-\frac{\varepsilon}{q}-1}}{(1+u)^{\lambda}} d u\right] d x \\
& =\int_{1}^{\infty} x^{-\varepsilon-1}\left[\int_{1 / x}^{1} \frac{u^{\lambda_{2}-\frac{\varepsilon}{q}-1}}{(1+u)^{\lambda}} d u\right] d x+\int_{1}^{\infty} x^{-\varepsilon-1}\left[\int_{1}^{\infty} \frac{u^{\lambda_{2}-\frac{\varepsilon}{q}-1}}{(1+u)^{\lambda}} d u\right] d x \\
& =\int_{0}^{1}\left(\int_{1 / u}^{\infty} x^{-\varepsilon-1} d x\right) \frac{u^{\lambda_{2}-\frac{\varepsilon}{q}-1}}{(1+u)^{\lambda}} d u+\frac{1}{\varepsilon} \int_{1}^{\infty} \frac{u^{\lambda_{2}-\frac{\varepsilon}{q}-1}}{(1+u)^{\lambda}} d u \\
& =\frac{1}{\varepsilon}\left[\int_{0}^{1} \frac{u^{\lambda_{2}+\frac{\varepsilon}{p}-1}}{(1+u)^{\lambda}} d u+\int_{1}^{\infty} \frac{u^{\lambda_{2}-\frac{\varepsilon}{q}-1}}{(1+u)^{\lambda}} d u\right] .
\end{aligned}
$$

So we obtain

$$
\int_{0}^{1} \frac{u^{\lambda_{2}+\frac{\varepsilon}{p}-1}}{(1+u)^{\lambda}} d u+\int_{1}^{\infty} \frac{u^{\lambda_{2}-\frac{\varepsilon}{q}-1}}{(1+u)^{\lambda}} d u \leq \varepsilon \tilde{I}<\varepsilon M \tilde{J}<\frac{M}{\left(\lambda_{1}-\frac{\varepsilon}{p}\right)\left(\lambda_{2}-\frac{\varepsilon}{q}\right)} .
$$

As $\varepsilon \rightarrow 0^{+}$in this inequality, in view of the continuity of the beta function, we find $B\left(\lambda_{1}, \lambda_{2}\right) \leq \frac{M}{\lambda_{1} \lambda_{2}}$, namely $\lambda_{1} \lambda_{2} B\left(\lambda_{1}, \lambda_{2}\right) \leq M$. Hence $M=\lambda_{1} \lambda_{2} B\left(\lambda_{1}, \lambda_{2}\right)$ is the best possible constant factor of (14).

The theorem is proved. 
Remark 1 We set $\hat{\lambda}_{1}:=\lambda_{1}+\frac{\lambda-\lambda_{1}-\lambda_{2}}{p}, \hat{\lambda}_{2}:=\lambda_{2}+\frac{\lambda-\lambda_{1}-\lambda_{2}}{q}$. It follows that $\hat{\lambda}_{1}+\hat{\lambda}_{2}=\lambda$. For $\lambda-\lambda_{1}-\lambda_{2} \in\left(-p \lambda_{1}, p\left(\lambda-\lambda_{1}\right)\right)$, we find

$$
\hat{\lambda}_{1}>\lambda_{1}+\frac{-p \lambda_{1}}{p}=0, \quad \hat{\lambda}_{1}<\lambda_{1}+\frac{p\left(\lambda-\lambda_{1}\right)}{p}=\lambda,
$$

namely, $0<\hat{\lambda}_{1}<\lambda$, and then $0<\hat{\lambda}_{2}<\lambda$. So we reduce (15) as follows:

$$
\begin{aligned}
I:= & \int_{0}^{\infty} \int_{0}^{\infty} \frac{f(x) g(y)}{(x+y)^{\lambda}} d x d y<\frac{\Gamma(\lambda+2)}{\Gamma(\lambda)} B^{\frac{1}{p}}\left(\lambda_{2}+1, \lambda+1-\lambda_{2}\right) B^{\frac{1}{q}}\left(\lambda_{1}+1, \lambda+1-\lambda_{1}\right) \\
& \times\left(\int_{0}^{\infty} x^{-p \hat{\lambda}_{1}-1} F^{p}(x) d x\right)^{\frac{1}{p}}\left(\int_{0}^{\infty} y^{-q \hat{\lambda}_{2}-1} G^{q}(y) d y\right)^{\frac{1}{q}}
\end{aligned}
$$

Theorem 2 If $\lambda-\lambda_{1}-\lambda_{2} \in\left(-p \lambda_{1}, p\left(\lambda-\lambda_{1}\right)\right)$ and the constant factor

$$
\frac{\Gamma(\lambda+2)}{\Gamma(\lambda)} B^{\frac{1}{p}}\left(\lambda_{2}+1, \lambda+1-\lambda_{2}\right) B^{\frac{1}{q}}\left(\lambda_{1}+1, \lambda+1-\lambda_{1}\right)
$$

in (18) is the best possible, then $\lambda_{1}+\lambda_{2}=\lambda$ with $\lambda_{1}, \lambda_{2} \in(0, \lambda)$.

Proof As regards to the assumptions, we find $0<\hat{\lambda}_{1}, \hat{\lambda}_{2}<\lambda$. By (16) the unified best possible constant factor in (18) must be of the form

$$
\hat{\lambda}_{1} \hat{\lambda}_{2} B\left(\hat{\lambda}_{1}, \hat{\lambda}_{2}\right)\left(=\frac{\Gamma(\lambda+2)}{\Gamma(\lambda)} B\left(\hat{\lambda}_{1}+1, \hat{\lambda}_{2}+1\right)\right)
$$

namely, it follows that

$$
B\left(\hat{\lambda}_{1}+1, \hat{\lambda}_{2}+1\right)=B^{\frac{1}{p}}\left(\lambda_{2}+1, \lambda+1-\lambda_{2}\right) B^{\frac{1}{q}}\left(\lambda_{1}+1, \lambda+1-\lambda_{1}\right) .
$$

By Hölder's inequality (see [27]) we obtain

$$
\begin{aligned}
B\left(\hat{\lambda}_{1}+1, \hat{\lambda}_{2}+1\right) & =\int_{0}^{\infty} \frac{u^{\left(\hat{\lambda}_{1}+1\right)-1}}{(1+u)^{\lambda+2}} d u \\
& =\int_{0}^{\infty} \frac{1}{(1+u)^{\lambda+2}} u^{\frac{\lambda+1-\lambda_{2}}{p}+\frac{\lambda_{1}+1}{q}-1} d u=\int_{0}^{\infty} \frac{1}{(1+u)^{\lambda+2}}\left(u^{\frac{\lambda-\lambda_{2}}{p}}\right)\left(u^{\frac{\lambda_{1}}{q}}\right) d u \\
& \leq\left[\int_{0}^{\infty} \frac{u^{\lambda-\lambda_{2}}}{(1+u)^{\lambda+2}} d u\right]^{\frac{1}{p}}\left[\int_{0}^{\infty} \frac{u^{\lambda_{1}}}{(1+u)^{\lambda+2}} d u\right]^{\frac{1}{q}} \\
& =B^{\frac{1}{p}}\left(\lambda_{2}+1, \lambda+1-\lambda_{2}\right) B^{\frac{1}{q}}\left(\lambda_{1}+1, \lambda+1-\lambda_{1}\right)
\end{aligned}
$$

We observe that (19) becomes equality if and only if there exist constants $A$ and $B$ such that they are not all zero and

$$
A u^{\lambda-\lambda_{2}}=B u^{\lambda_{1}} \quad \text { a.e. in } R_{+}
$$

(see [26]). Without loss of generality, we suppose $A \neq 0$. It follows that $u^{\lambda-\lambda_{2}-\lambda_{1}}=\frac{B}{A}$ a.e. in $R_{+}$, namely, $\lambda-\lambda_{1}-\lambda_{2}=0$, and then $\lambda_{1}+\lambda_{2}=\lambda$ with $\lambda_{1}, \lambda_{2} \in(0, \lambda)$.

The theorem is proved. 
Theorem 3 The following statements are equivalent:

(i) $B^{\frac{1}{p}}\left(\lambda_{2}+1, \lambda+1-\lambda_{2}\right) B^{\frac{1}{q}}\left(\lambda_{1}+1, \lambda+1-\lambda_{1}\right)$ is independent of $p, q$;

(ii) $B^{\frac{1}{p}}\left(\lambda_{2}+1, \lambda+1-\lambda_{2}\right) B^{\frac{1}{q}}\left(\lambda_{1}+1, \lambda+1-\lambda_{1}\right)$ is expressible as a single integral;

(iii) If $\lambda-\lambda_{1}-\lambda_{2} \in\left(-p \lambda_{1}, p\left(\lambda-\lambda_{1}\right)\right)$, then $\lambda_{1}+\lambda_{2}=\lambda\left(\lambda_{1}, \lambda_{2} \in(0, \lambda)\right)$;

(iv) The constant factor

$$
\frac{\Gamma(\lambda+2)}{\Gamma(\lambda)} B^{\frac{1}{p}}\left(\lambda_{2}+1, \lambda+1-\lambda_{2}\right) B^{\frac{1}{q}}\left(\lambda_{1}+1, \lambda+1-\lambda_{1}\right)
$$

in (15) is the best possible.

Proof (i) $\Rightarrow$ (ii). Since $B^{\frac{1}{p}}\left(\lambda_{2}+1, \lambda+1-\lambda_{2}\right) B^{\frac{1}{q}}\left(\lambda_{1}+1, \lambda+1-\lambda_{1}\right)$ is independent of $p$, $q$, we find

$$
\begin{aligned}
& B^{\frac{1}{p}}\left(\lambda_{2}+1, \lambda+1-\lambda_{2}\right) B^{\frac{1}{q}}\left(\lambda_{1}+1, \lambda+1-\lambda_{1}\right) \\
& \quad=\lim _{p \rightarrow \infty} \lim _{q \rightarrow 1^{+}} B^{\frac{1}{p}}\left(\lambda_{2}+1, \lambda+1-\lambda_{2}\right) B^{\frac{1}{q}}\left(\lambda_{1}+1, \lambda+1-\lambda_{1}\right) \\
& \quad=B\left(\lambda_{1}+1, \lambda+1-\lambda_{1}\right)=\int_{0}^{\infty} \frac{u^{\lambda_{1}}}{(1+u)^{\lambda+2}} d u,
\end{aligned}
$$

which is a single integral.

(ii) $\Rightarrow$ (iii). Suppose that $B^{\frac{1}{p}}\left(\lambda_{2}+1, \lambda+1-\lambda_{2}\right) B^{\frac{1}{q}}\left(\lambda_{1}+1, \lambda+1-\lambda_{1}\right)$ is expressible as a single integral $\int_{0}^{\infty} \frac{1}{(1+u)^{\lambda+2}} u^{\frac{\lambda+1-\lambda_{2}}{p}+\frac{\lambda_{1}+1}{q}-1} d u$. Then (19) keeps the form of equality. By the proof of Theorem 2 we have $\lambda_{1}+\lambda_{2}=\lambda\left(\lambda_{1}, \lambda_{2} \in(0, \lambda)\right)$.

(iii) $\Rightarrow$ (iv). If $\lambda_{1}+\lambda_{2}=\lambda\left(\lambda_{1}, \lambda_{2} \in(0, \lambda)\right)$, then by Theorem 1 the constant factor

$$
\frac{\Gamma(\lambda+2)}{\Gamma(\lambda)} B^{\frac{1}{p}}\left(\lambda_{2}+1, \lambda+1-\lambda_{2}\right) B^{\frac{1}{q}}\left(\lambda_{1}+1, \lambda+1-\lambda_{1}\right)\left(=\lambda_{1} \lambda_{2} B\left(\lambda_{1}, \lambda_{2}\right)\right)
$$

in (13) is the best possible.

(iv) $\Rightarrow$ (i). In this case, by Theorem 2 we have $\lambda_{1}+\lambda_{2}=\lambda$, and

$$
B^{\frac{1}{p}}\left(\lambda_{2}+1, \lambda+1-\lambda_{2}\right) B^{\frac{1}{q}}\left(\lambda_{1}+1, \lambda+1-\lambda_{1}\right)=B\left(\lambda_{1}+1, \lambda_{2}+1\right)
$$

is independent of $p, q$.

Hence statements (i), (ii), (iii), and (iv) are equivalent.

The theorem is proved.

Remark 2 If $\mu+\sigma=s(\mu, \sigma \in(0, s))$, then inequality (11) reduces to

$$
\begin{aligned}
\int_{0}^{\infty} \int_{0}^{\infty} \frac{f(x) g(y)}{(x+y)^{s}} d x d y \leq & B(\mu, \sigma)\left[\int_{0}^{\infty} x^{p(1-\mu)-1} f^{p}(x) d x\right]^{\frac{1}{p}} \\
\times & {\left[\int_{0}^{\infty} y^{q(1-\sigma)-1} g^{q}(y) d y\right]^{\frac{1}{q}} }
\end{aligned}
$$

We confirm that the constant factor $B(\mu, \sigma)$ in (20) is the best possible. Otherwise, we would reach a contradiction by (17) that the constant factor in (16) is not the best possible. 
Replacing $x$ by $\frac{1}{x}$ and then $x^{s-2} f\left(\frac{1}{x}\right)$ by $f(x)$ in (20), we have the following Hardy-Hilbert's integral inequality with a nonhomogeneous kernel and the best possible constant factor $B(s-\sigma, \sigma)$ :

$$
\begin{aligned}
\int_{0}^{\infty} \int_{0}^{\infty} \frac{f(x) g(y)}{(1+x y)^{s}} d x d y \leq & B(s-\sigma, \sigma)\left[\int_{0}^{\infty} x^{p(1-\sigma)-1} f^{p}(x) d x\right]^{\frac{1}{p}} \\
\times & {\left[\int_{0}^{\infty} y^{q(1-\sigma)-1} g^{q}(y) d y\right]^{\frac{1}{q}} }
\end{aligned}
$$

\section{A corollary and some particular cases}

Replacing $x$ by $\frac{1}{x}$ in (15) and setting $\hat{f}(x)=x^{\lambda-2} f\left(\frac{1}{x}\right)$, we define

$$
F_{\lambda}(x):=\int_{\frac{1}{x}}^{\infty} t^{-\lambda} \hat{f}(t) d t\left(=\int_{\frac{1}{x}}^{\infty} f\left(\frac{1}{u}\right) \frac{1}{u^{2}} d u=\int_{0}^{x} f(t) d t\right)
$$

Then replacing $\hat{f}(x)$ by $f(x)$, we have $F_{\lambda}(x)=\int_{\frac{1}{x}}^{\infty} t^{-\lambda} f(t) d t$ and the following Hilbert-type integral inequality with nonhomogeneous kernel:

$$
\begin{aligned}
\int_{0}^{\infty} \int_{0}^{\infty} \frac{f(x) g(y)}{(1+x y)^{\lambda}} d x d y< & \frac{\Gamma(\lambda+2)}{\Gamma(\lambda)} B^{\frac{1}{p}}\left(\lambda_{2}+1, \lambda+1-\lambda_{2}\right) B^{\frac{1}{q}}\left(\lambda_{1}+1, \lambda+1-\lambda_{1}\right) \\
& \times\left[\int_{0}^{\infty} x^{-p \lambda_{1}-\left(\lambda-\lambda_{1}-\lambda_{2}\right)-1} F_{\lambda}^{p}(x) d x\right]^{\frac{1}{p}} \\
& \times\left[\int_{0}^{\infty} y^{-q \lambda_{2}-\left(\lambda-\lambda_{1}-\lambda_{2}\right)-1} G^{q}(y) d y\right]^{\frac{1}{q}}
\end{aligned}
$$

which is equivalent to (15).

In view of Theorem 3, we have the following:

Corollary 1 Assuming that $\lambda-\lambda_{1}-\lambda_{2} \in\left(-p \lambda_{1}, p\left(\lambda-\lambda_{1}\right)\right)$, the constant factor

$$
\frac{\Gamma(\lambda+2)}{\Gamma(\lambda)} B^{\frac{1}{p}}\left(\lambda_{2}+1, \lambda+1-\lambda_{2}\right) B^{\frac{1}{q}}\left(\lambda_{1}+1, \lambda+1-\lambda_{1}\right)
$$

in (22) is the best possible if and only if $\lambda_{1}+\lambda_{2}=\lambda\left(\lambda_{1}, \lambda_{2} \in(0, \lambda)\right)$.

In the case of $\lambda_{1}+\lambda_{2}=\lambda$, (22) reduces to the following Hilbert-type integral inequality with nonhomogeneous kernel and the best possible constant factor $\lambda_{1} \lambda_{2} B\left(\lambda_{1}, \lambda_{2}\right)$ :

$$
\begin{aligned}
\int_{0}^{\infty} \int_{0}^{\infty} \frac{f(x) g(y)}{(1+x y)^{\lambda}} d x d y< & \lambda_{1} \lambda_{2} B\left(\lambda_{1}, \lambda_{2}\right) \\
& \times\left\{\int_{0}^{\infty} x^{-p \lambda_{1}-1} F_{\lambda}^{p}(x) d x\right\}^{\frac{1}{p}}\left\{\int_{0}^{\infty} y^{-q \lambda_{2}-1} G^{q}(y) d y\right\}^{\frac{1}{q}},
\end{aligned}
$$

which is equivalent to (16). 
Remark 3 In (16) and (23), for $\lambda_{1}=\frac{\lambda}{q}, \lambda_{2}=\frac{\lambda}{p}$, we have the following equivalent inequalities:

$$
\begin{aligned}
\int_{0}^{\infty} \int_{0}^{\infty} \frac{f(x) g(y)}{(x+y)^{\lambda}} d x d y< & \frac{\lambda^{2}}{p q} B\left(\frac{\lambda}{p}, \frac{\lambda}{q}\right) \\
& \times\left(\int_{0}^{\infty} x^{(1-p) \lambda-1} F^{p}(x) d x\right)^{\frac{1}{p}} \\
& \times\left(\int_{0}^{\infty} y^{(1-q) \lambda-1} G^{q}(y) d y\right)^{\frac{1}{q}}, \\
\int_{0}^{\infty} \int_{0}^{\infty} \frac{f(x) g(y)}{(1+x y)^{\lambda}} d x d y< & \frac{\lambda^{2}}{p q} B\left(\frac{\lambda}{p}, \frac{\lambda}{q}\right) \\
& \times\left(\int_{0}^{\infty} x^{(1-p) \lambda-1} F_{\lambda}^{p}(x) d x\right)^{\frac{1}{p}} \\
& \times\left(\int_{0}^{\infty} y^{(1-q) \lambda-1} G^{q}(y) d y\right)^{\frac{1}{q}},
\end{aligned}
$$

and for $\lambda_{1}=\frac{\lambda}{p}, \lambda_{2}=\frac{\lambda}{q}$, we have the following equivalent inequalities:

$$
\begin{aligned}
\int_{0}^{\infty} \int_{0}^{\infty} \frac{f(x) g(y)}{(x+y)^{\lambda}} d x d y< & \frac{\lambda^{2}}{p q} B\left(\frac{\lambda}{p}, \frac{\lambda}{q}\right) \\
& \times\left(\int_{0}^{\infty} x^{-\lambda-1} F^{p}(x) d x\right)^{\frac{1}{p}}\left(\int_{0}^{\infty} y^{-\lambda-1} G^{q}(y) d y\right)^{\frac{1}{q}} \\
\int_{0}^{\infty} \int_{0}^{\infty} \frac{f(x) g(y)}{(1+x y)^{\lambda}} d x d y & <\frac{\lambda^{2}}{p q} B\left(\frac{\lambda}{p}, \frac{\lambda}{q}\right) \\
& \times\left(\int_{0}^{\infty} x^{-\lambda-1} F_{\lambda}^{p}(x) d x\right)^{\frac{1}{p}}\left(\int_{0}^{\infty} y^{-\lambda-1} G^{q}(y) d y\right)^{\frac{1}{q}} .
\end{aligned}
$$

In particular, for $p=q=2$, both inequalities (24) and (26) reduce to

$$
\begin{aligned}
\int_{0}^{\infty} \int_{0}^{\infty} \frac{f(x) g(y)}{(x+y)^{\lambda}} d x d y< & \frac{\lambda^{2}}{4} B\left(\frac{\lambda}{2}, \frac{\lambda}{2}\right) \\
& \times\left(\int_{0}^{\infty} x^{-\lambda-1} F^{2}(x) d x \int_{0}^{\infty} y^{-\lambda-1} G^{2}(y) d y\right)^{\frac{1}{2}}
\end{aligned}
$$

and both (25) and (27) reduce to the following equivalent form of (25):

$$
\begin{aligned}
\int_{0}^{\infty} \int_{0}^{\infty} \frac{f(x) g(y)}{(1+x y)^{\lambda}} d x d y< & \frac{\lambda^{2}}{4} B\left(\frac{\lambda}{2}, \frac{\lambda}{2}\right) \\
& \times\left(\int_{0}^{\infty} x^{-\lambda-1} F_{\lambda}^{2}(x) d x \int_{0}^{\infty} y^{-\lambda-1} G^{2}(y) d y\right)^{\frac{1}{2}} .
\end{aligned}
$$

The constant factors in the inequalities of Remark 3 are the best possible.

\section{Conclusions}

In this paper, following [21, 22], using the weight functions and the idea of introduced parameters, we give a new Hilbert-type integral inequality with the kernel $\frac{1}{(x+y)^{\lambda}}(\lambda>0)$ 
involving the upper limit functions and the beta and gamma functions (Theorem 1). The preliminaries and the equivalent statements of the best possible constant factor related to a few parameters are considered in Theorems 2 and 3. As applications, we obtain a corollary in the case of nonhomogeneous kernel and some particular inequalities (Corollary 1 and Remark 3). The lemmas and theorems provide an extensive account of inequalities of this type.

\author{
Acknowledgements \\ The authors thank the referee for his useful proposal to reform the paper.
}

Funding

This work is supported by the National Natural Science Foundation (No. 61772140), Natural Science Foundation of Jishou University (No. Jd16012), and Science and Technology Planning Project Item of Guangzhou City (No. 201707010229). We are grateful for their help.

\title{
Competing interests
}

The authors declare that they have no competing interests.

\section{Authors' contributions}

BY carried out the mathematical studies, participated in the sequence alignment, and drafted the manuscript. HM participated in the design of the study and performed the numerical analysis. Both authors read and approved the final manuscript.

\section{Author details}

${ }^{1}$ College of Mathematics and Statistics, Jishou University, Jishou, P.R. China. ${ }^{2}$ Department of Mathematics, Guangdong University of Education, Guangzhou, China.

\section{Publisher's Note}

Springer Nature remains neutral with regard to jurisdictional claims in published maps and institutional affiliations.

Received: 9 August 2019 Accepted: 29 December 2019 Published online: 09 January 2020

\section{References}

1. Hardy, G.H., Littlewood, J.E., Polya, G.: Inequalities. Cambridge University Press, Cambridge (1934)

2. Yang, B.C.: The Norm of Operator and Hilbert-Type Inequalities. Science Press, Beijing (2009)

3. Yang, B.C.: Hilbert-Type Integral Inequalities. Bentham Science Publishers, The United Arab Emirates (2009)

4. Yang, B.C.: On the norm of an integral operator and applications. J. Math. Anal. Appl. 321, 182-192 (2006)

5. Xu, J.S.: Hardy-Hilbert's inequalities with two parameters. Adv. Math. 36(2), 63-76 (2007)

6. Yang, B.C.: On the norm of a Hilbert's type linear operator and applications. J. Math. Anal. Appl. 325, 529-541 (2007)

7. Xie, Z.T., Zeng, Z., Sun, Y.F.: A new Hilbert-type inequality with the homogeneous kernel of degree -2 . Adv. Appl. Math. Sci. 12(7), 391-401 (2013)

8. Zhen, Z., Raja Rama Gandhi, K., Xie, Z.T.: A new Hilbert-type inequality with the homogeneous kernel of degree -2 and with the integral. Bull. Math. Sci. Appl. 3(1), 11-20 (2014)

9. Xin, D.M.: A Hilbert-type integral inequality with the homogeneous kernel of zero degree. Math. Theory Appl. 30(2), 70-74 (2010)

10. Azar, L.E.: The connection between Hilbert and Hardy inequalities. J. Inequal. Appl. 2013, 452 (2013)

11. Batbold, T., Sawano, Y.: Sharp bounds for $m$-linear Hilbert-type operators on the weighted Morrey spaces. Math. Inequal. Appl. 20, 263-283 (2017)

12. Adiyasuren, V., Batbold, T., Krnic, M.: Multiple Hilbert-type inequalities involving some differential operators. Banach J. Math. Anal. 10, 320-337 (2016)

13. Adiyasuren, V., Batbold, T., Krni'c, M.: Hilbert-type inequalities involving differential operators, the best constants and applications. Math. Inequal. Appl. 18, 111-124 (2015)

14. Rassias, M.Th., Yang, B.C.: On half-discrete Hilbert's inequality. Appl. Math. Comput. 220, 75-93 (2013)

15. Yang, B.C., Krnic, M.: A half-discrete Hilbert-type inequality with a general homogeneous kernel of degree 0. J. Math. Inequal. 6(3), 401-417 (2012)

16. Rassias, M.Th., Yang, B.C.: A multidimensional half-discrete Hilbert-type inequality and the Riemann zeta function. Appl. Math. Comput. 225, 263-277 (2013)

17. Rassias, M.Th., Yang, B.C.: On a multidimensional half-discrete Hilbert-type inequality related to the hyperbolic cotangent function. Appl. Math. Comput. 242, 800-813 (2013)

18. Huang, Z.X., Yang, B.C.: On a half-discrete Hilbert-type inequality similar to Mulholland's inequality. J. Inequal. Appl. 2013, $290(2013)$

19. Yang, B.C., Lebnath, L.: Half-Discrete Hilbert-Type Inequalities. World Scientific Publishing, Singapore (2014)

20. Krnic, M., Pecaric, J.: Extension of Hilbert's inequality. J. Math. Anal. Appl. 324(1), 150-160 (2006)

21. Adiyasuren, V., Batbold, T., Azar, L.E.: A new discrete Hilbert-type inequality involving partial sums. J. Inequal. Appl. 2019, 127 (2019)

22. Hong, Y., Wen, Y.: A necessary and sufficient condition of that Hilbert type series inequality with homogeneous kernel has the best constant factor. Ann. Math. 37A(3), 329-336 (2016) 
23. Hong, Y.: On the structure character of Hilbert's type integral inequality with homogeneous kernel and applications. J. Jilin Univ. Sci. Ed. 55(2), 189-194 (2017)

24. Hong, Y., Huang, Q.L., Yang, B.C., Liao, J.L.: The necessary and sufficient conditions for the existence of a kind of Hilbert-type multiple integral inequality with the non-homogeneous kernel and its applications. J. Inequal. Appl. 2017, 316 (2017)

25. Xin, D.M., Yang, B.C., Wang, A.Z.: Equivalent property of a Hilbert-type integral inequality related to the beta function in the whole plane. J. Funct. Spaces 2018, Article ID 2691816 (2018)

26. Hong, Y., He, B., Yang, B.C.: Necessary and sufficient conditions for the validity of Hilbert type integral inequalities with a class of quasi-homogeneous kernels and its application in operator theory. J. Math. Inequal. 12(3), 777-788 (2018)

27. Kuang, J.C.: Applied Inequalities. Shangdong Science and Technology Press, Jinan (2004)

28. Kuang, J.C.: Real and Functional Analysis (Continuation), vol. 2. Higher Education Press, Beijing (2015)

\section{Submit your manuscript to a SpringerOpen ${ }^{\circ}$} journal and benefit from:

- Convenient online submission

Rigorous peer review

- Open access: articles freely available online

- High visibility within the field

- Retaining the copyright to your article

Submit your next manuscript at $\boldsymbol{\Delta}$ springeropen.com 